\title{
Gain Control in EDFA's by Pump Compensation
}

\author{
L. Tančevski, L. A. Rusch, and A. Bononi
}

\begin{abstract}
Gain control by means of pump compensation in cascades of erbium-doped fiber amplifiers (EDFA's) is analyzed. Based on analytical expressions for the 1-dB rise/fall time for the case of channels add/drop, required pump power changes at each amplifier are derived in the form of simple closed expressions. Numerical simulations of a system comprising 20 wavelengthdivision-multiplexed channels transmitted through a cascade of 35 EDFA's are performed demonstrating the effectiveness of the gain control.
\end{abstract}

Index Terms - Doped-fiber amplifiers, gain compensation, optical communications.

\section{INTRODUCTION}

$\mathbf{R}$ ECENTLY, there has been growing interest in evaluating the time responses of erbium-doped fiber amplifiers (EDFA's) in the case of a channel drop or add in a wavelengthdivision-multiplexing (WDM) network scenario [1], [2]. Following the analysis in [1] we first develop simple analytical expressions for the time responses expressed through the figure of merit $1 \mathrm{~dB}$ time, i.e., the time in which the power excursion reaches $1 \mathrm{~dB}$ [2]. Building on the derivations for the 1-dB time, we next focus on analyzing a gain control mechanism based on pump compensation. Pump power is adjusted to compensate the slope of the power excursion at each amplifier. Required pump power changes are derived in a very simple closed form. In order to investigate the effectiveness of the scheme, numerical simulations of a system comprising 20 WDM channels fed to a cascade of 35 EDFA's were performed and the resulting power and signal-to-noiseratio (SNR) excursions with and without pump compensation are presented. The results indicate an effective compensation scheme limiting the excursions to within a predetermined margin. An experimental demonstration of gain control using pump compensation was reported in [3].

\section{DERIVATION OF THE ANALYTICAL EXPRESSIONS}

Define

$$
\Delta P_{k}^{\text {out }}(t)=\frac{P_{k}^{\operatorname{in}}(t) e^{G_{k}(t)}}{P_{k}^{\operatorname{in}}(0) e^{G_{k}(0)}}=\Delta P_{k}^{\operatorname{in}}(t) e^{G_{k}(t)-G_{k}(0)}
$$

Manuscript received February 5, 1998; revised April 23, 1998. This work was supported by a grant from the Natural Sciences and Engineering Research Council of Canada and by QuèbecTel.

L. Tančevski is with Alcatel Network Systems, Corporate Research Center, Richardson, TX 75081 USA.

L. A. Rusch is with the Laval University, Department of Electrical and Computer Engineering, Quèbec, PQ, G1K-7P4, Canada.

A. Bononi is with the Università di Parma, Dipartimento di Ingegneria dell'Informazione, I-43100 Parma, Italy.

Publisher Item Identifier S 1041-1135(98)06277-6. to be the power excursion of the output power of $k$ th channel relative to the steady state and due to the step transition of the signal channels at $t=0^{+}$, where $G_{k}$ is the gain given in natural logarithm units. Then

$$
\begin{aligned}
& \left.\frac{d}{d t}\left(\ln \Delta P_{k}^{\text {out }}(t)\right)\right|_{t=0^{+}} \\
& \quad=\left.\frac{d}{d t}\left(\ln \Delta P_{k}^{\text {in }}(t)\right)\right|_{t=0^{+}}+\left.\frac{d}{d t} G_{k}(t)\right|_{t=0^{+}}
\end{aligned}
$$

represents the slope of the output power excursion in natural logarithm units. Since the interamplifier loss is independent of time, (2) gives the relation between the output power slope of the amplifier and the output power slope of the previous amplifier in the chain

$$
\Delta_{k}^{(i)}-\Delta_{k}^{(i-1)}=\left.\frac{d}{d t} G_{k}^{(i)}\right|_{t=0^{+}}, \quad \Delta_{k}^{(0)}=0
$$

where $i$ designates the $i$ th amplifier in the chain. This is a difference equation describing the slope evolution along the cascade with initial condition that the slope of the input power at the first amplifier is zero. Assuming the system is in steady state before the step power transition, from [1, eq. (8)]

$$
\begin{aligned}
\frac{d}{d t} G_{k}^{(i)}(t) & =\sum_{j=0}^{N} \frac{P_{j}^{(i)}\left(0^{+}\right)-P_{j}^{(i)}\left(0^{-}\right)}{\tau P_{k}^{\mathrm{IS}}}\left(e^{G_{j}^{(i)}(0)}-1\right) \\
& =\sum_{j=0}^{N} \frac{\Delta P_{j}^{(i)}}{\tau P_{k}^{\mathrm{IS}}}\left(e^{G_{j}^{(i)}(0)}-1\right) \\
\Delta P_{j}^{(i)} & =\Delta P_{j}^{(i-1)} e^{G_{j}^{(i-1)}-\operatorname{Loss}(j)}, \quad \Delta P_{j}^{(1)}=\Delta P_{j}
\end{aligned}
$$

where $\tau$ is the fluorescence time, $\Delta P_{j}^{(i)}$ is the power change in photons/s as seen by the $i$ th amplifier, $P_{k}^{\mathrm{IS}}$ is the intrinsic saturation power in photons/s, $N$ is the number of signal channels and $\operatorname{Loss}(j)$ is the interamplifier loss including the effect of any notch filter for gain equalization. The summation goes over all the channels - it should be understood that for channels not experiencing step transition $\Delta P_{j}(i)=0$. We now approximate the output power excursion by a linear Taylor series expansion

$$
\begin{aligned}
& \ln \Delta P_{k}^{\text {out }}(t) \\
& \left.\quad \doteq \ln \Delta P_{k}^{\text {out }}(t)\right|_{t=0^{+}}+\left.t \frac{d}{d t}\left(\ln \Delta P_{k}^{\text {out }}(t)\right)\right|_{t=0^{+}}=t \Delta_{k}^{(i)} .
\end{aligned}
$$

Making the transition from natural to decimal logarithms, the $1-\mathrm{dB}$ rise or fall time is given by $t_{1 \mathrm{~dB}}^{(i)}=\ln 10 /\left(10 \Delta_{k}^{(i)}\right)$.

The knowledge of the slope evolution along the cascade and the associated $1-\mathrm{dB}$ times could be put into practical use in 
designing a gain control mechanism by switching the pump. In this scenario, the pump is switched after a certain amount of time has elapsed since the power transition. Guaranteeing $1-\mathrm{dB}$ power excursions to a tolerable performance entails knowledge of the fastest 1-dB rise/fall time-the pump must be switched within this time period. The amount of pump power change should be such that it provides an effective gain control—knowledge of the slope could be employed to design a pump compensation scheme that exactly counter-measures the slope by inducing an additional slope with the opposite sign. Hence, from (4), the pump change $\Delta P_{0}^{(i)}$ given in units of photons/sec necessary to compensate the slope at the $i$ th amplifier is given by ${ }^{1}$

$$
\Delta P_{0}^{(i)} \doteq-\frac{1}{e^{G_{0}^{(i)}(0)}-1} \sum_{j=1}^{N} \Delta P_{j}^{(i)}\left(e^{G_{j}^{(i)}(0)}-1\right) .
$$

Some comments are in order for the $\doteq \operatorname{sign}$ in (6): if the pump change occurs at $t=0^{+},(6)$ gives an exact result. In reality, it will occur after some time necessary to detect the power change and to align the pump power. In the linear regime of the power excursion (5), the slope at $t=t_{1}>0^{+}$will be slightly less than the slope at time $t=0^{+}$, easily deduced from the convexity of the power excursion curve. Hence, the pump induced compensation slope at $t=t_{1}$ will be slightly stronger than the signal power change slope induced at $t=0^{+}$ and will gradually drive the power excursion back to the zero level. Although the derivation is applied to cascades of singlestage EDFA's, cascades of $m$-stage EDFA's [9] can be treated in a similar manner. Since an $m$-stage EDFA comprises a chain of $m$ single-stage EDFA's with some additional losses and possibly an isolator to block the backward-propagating amplified spontaneous emission (ASE) between stages, (6) can be applied to each single-stage EDFA within the $m$-stage amplifier.

\section{NUMERICAL SIMULATIONS}

In order to check the validity of the derived expressions, we performed numerical simulations. Following the analysis in [7], the system under consideration comprises $20 \mathrm{WDM}$ channels, spaced uniformly between 1542 and $1551.5 \mathrm{~nm}$ with 0.5 -nm spacing and each carrying $-10-\mathrm{dBm}$ input power. Such traffic was fed into a cascade of 35 EDFA's (the spectral parameters of the EDFA's were taken from [4]). The amplifier length was $14.7 \mathrm{~m}$ and the pump power was $18.4 \mathrm{dBm}$ giving roughly $10-\mathrm{dB}$ gain to exactly balance the interamplifier loss. To assure gain equalization, a notch filter with filter depth of $1.4 \mathrm{~dB}$, central wavelength of $1546 \mathrm{~nm}$ and bandwidth of 2.5 $\mathrm{nm}$ was placed periodically after every 4 amplifiers [7], [8] and a filter was placed after every EDFA to block the harmful ASE below $1540 \mathrm{~nm}$ [7]. An optical filter of width $0.125 \mathrm{~nm}$ was assumed at the detector. The optical SNR at the output of the first, tenth, twentieth and the 35th EDFA for the steadystate case are plotted in Fig. 1(a) indicating fairly flat SNR over the channels (represented with circles).

\footnotetext{
${ }^{1}$ This expression was derived differently in [9].
}

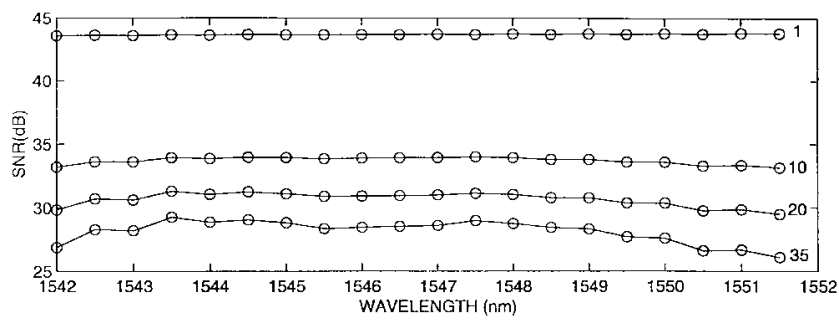

(a)

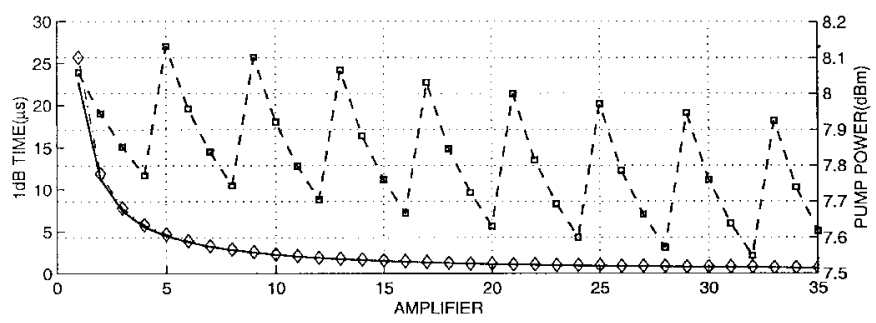

(b)

Fig. 1. Simulation of a cascade of 35 amplifiers: (a) optical SNR at the output of the 1st, 10th, 20th, and 35th amplifier, (b) 1-dB times and pump compensation powers for 19-channel drop. Full line-analytically calculated 1-dB times, broken line and diamonds-numerically calculated times, broken line and boxes-pump power.

Calculations of the transients were based on the model introduced in [1] into which ASE was included as outlined in [6] and [5]. The resulting ordinary differential equation (ODE) was solved in $0.1-\mu$ s time increments. First, 19 out of the possible 20 channels were dropped (the surviving channel was the first channel at $1542 \mathrm{~nm}$ ). We next calculated the $1-\mathrm{dB}$ times, the pump values for compensation and the power and SNR excursions, and then, after $400 \mu \mathrm{s}$, the channels were reinstated and the calculations repeated. Fig. 1(b) summarizes the 1-dB times calculated analytically using (5) (full line), the 1 $\mathrm{dB}$ times calculated numerically by solving the ODE (broken line with diamonds) and the pump power for compensation calculated using (6) (broken line with boxes). Note the excellent agreement between the analytical and numerical $1 \mathrm{~dB}$ times. Also note the sawtooth character of the pump curve, a clear consequence of the notch filters being inserted after every four EDFA's. The resulting power and optical SNR excursions are depicted in Fig. 2(a) and (b), respectively, representing the output of the first, tenth, twentieth and the 35th EDFA. Excursions as large as $18 \mathrm{~dB}$ are observed.

Having calculated the pump values for compensation and the 1-dB times, a second run was performed with pump compensation. The initial pump values were $18.4 \mathrm{dBm}$, but after $0.6 \mu \mathrm{s}$ (the fastest 1-dB time in the cascade) the pumps were switched to the newly calculated values. The resulting power and optical SNR excursions are depicted in Fig. 2(c) and (d), respectively. Initially, the excursion goes as in the uncompensated case, but when the pump is switched a negative slope is induced which starts driving the excursion back to the zero level as expected. This is more pronounced further along the cascade, as $0.6 \mu$ s is closer to the limit of the linear regime. Hence, the slope induced by the pump change is stronger than the original slope and the rate with which the excursion decays is faster. For the amplifiers closer to the beginning 


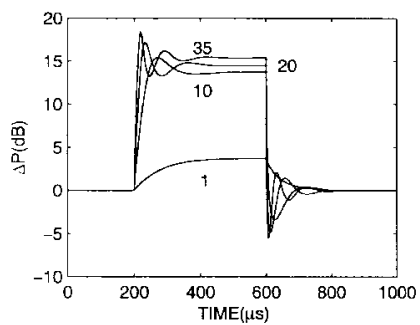

(a)

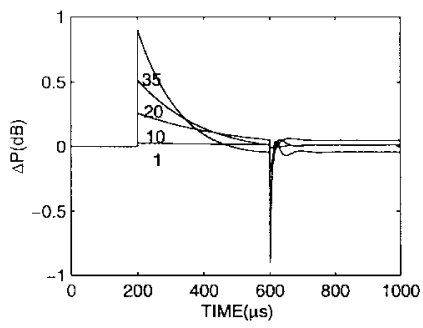

(c)

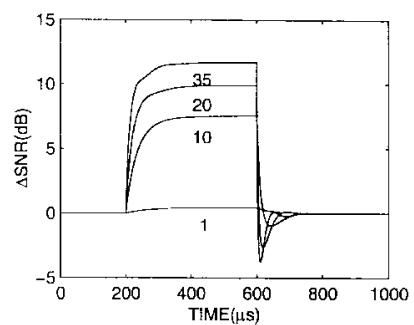

(b)

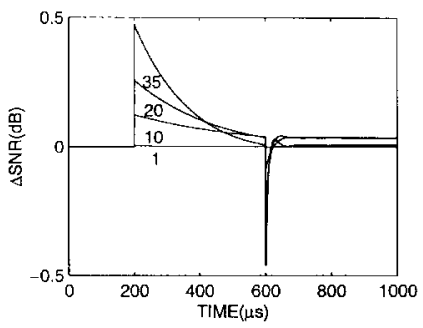

(d)
Fig. 2. Simulation of dropping and then adding back 19 channels in a cascade of 35 amplifiers. (a) Power excursion of the surviving channel. (b) SNR excursion. (c) Power excursion with pump compensation. (d) SNR excursion with pump compensation. Curves apply to the first, tenth, 20th, and the 35th amplifier.

of the cascade, $0.6 \mu \mathrm{s}$ is well within the linear regime and hence the additional slope is much closer to the originally induced slope - the rate of decay toward the zero level is much slower. Note that the largest power change occurs at the output of the 35th amplifier and is approximately $0.9 \mathrm{~dB}$, in good agreement with the analytical results that indicate $0.64-\mu \mathrm{s} 1$ $\mathrm{dB}$ rise time for the 35 th amplifier. A practical implementation of the gain control would require tapping off some light and detecting the power of the signal channels; this detection is particularly straightforward as we only need to identify a step transition in the power. Since the gain is kept constant at all times, a simple but fast electronic circuit can compute required pump power transition given by (6), after which the pump power can be suitably adjusted. Detecting the light, calculating the required pump transition, and (above all) adjusting the pump power involves an additional time factor, which can be effectively compensated by inserting a "look-ahead" delay in the signal path. This leads to "near-coincidence" of the signal and pump induced change on a time-scale shorter than the 0.6 $\mu$ s considered in the numerical simulations.

\section{REFERENCES}

[1] Y. Sun, G. Luo, J. L. Zyskind, A. A. M. Saleh, A. K. Srivastava, and J. W. Sulhoff, "Model for gain dynamics in erbium-doped fiber amplifiers," Electron. Lett., vol. 32, no. 16, pp. 1490-1491, Aug. 1996.

[2] Y. Sun, A. K. Srivastava, J. L. Zyskind, J. W. Sulhoff, C. Wolf, and R. W. Tkach, "Fast power transients in WDM optical networks with cascaded EDFA's," Electron. Lett., vol. 33, no. 4, pp. 313-314, Feb. 1997.

[3] A. K. Srivastava, Y. Sun, J. L. Zyskind, J. W. Sulhoff, C. Wolf, and R. W. Tkach, "Fast gain control in an erbium-doped fiber amplifiers," in Proc. Optical Amplifiers and Their Applications'96, Monterey, CA, July 1996, postdeadline paper, PDP4.

[4] E. Desurvire, Erbium-Doped Fiber Amplifiers. New York: Wiley, 1994.

[5] T. Georges and E. Delevaque, "Analytic modeling of high-gain erbiumdoped fiber amplifiers," Opt. Lett., vol. 17, no. 16, pp. 1113-1115, Aug. 1992.

[6] A. Bononi, L. Barbieri, and L. A. Rusch, "Using SPICE to simulate gain dynamics in doped-fiber amplifier chains," presented at OFC'98, Feb. 1998.

[7] W. Zeiler, F. Di Pasquale, P. Bayvel, and J. E. Midwinter, "Modeling of four-wave mixing and gain peaking in amplified WDM optical communication systems and networks," J. Lightwave Technol., vol. 14, pp. 1933-1942, Sept. 1996.

[8] A. E. Willner and S. Hwang, "Transmission of many WDM channels through a cascade of EDFA's in long-distance links and ring networks," J. Lightwave Technol., vol. 13, pp. 802-816, May 1995.

[9] Y. Sun, J. L. Zyskind, and A. K. Srivastava, "Average inversion level, modeling, and physics of erbium-doped fiber amplifiers," IEEE J. Select. Topics Quantum Electron., vol. 3, pp. 991-1004, Aug. 1997. 